\title{
COMMENTARY
}

\section{The first step in utilizing immune-modulating therapies: immune status determination}

\author{
Charles C Caldwell*1 and Richard S Hotchkiss ${ }^{2}$ \\ See related research by Oiva et al., http://ccforum.com/content/14/6/R207
}

\begin{abstract}
Recently, a single center study conducted by Oiva and coworkers and published in Critical Care demonstrated that phospho-specific whole blood flow cytometry could be used to assess activated signaling pathways in leukocytes isolated from pancreatitis patients. The authors demonstrated that this methodology had the potential to determine the current status of a patient's immune state. Although the experimental cohort was clinically homogeneous, the observed data were heterogeneous. Altogether, these results suggest that prior to administering immune-modulatory therapies in inflammatory diseases, it will be beneficial to first determine immune status. Rapid results from whole blood phospho-specific flow cytometry may allow for determination of immune status, improve early diagnosis, and provide a rational basis for immunomodulatory therapies.
\end{abstract}

Recently, a single center study conducted by Oiva and coworkers and published in Critical Care [1] demonstrated that phospho-specific whole blood flow cytometry could be used to assess activated signaling pathways in leukocytes isolated from pancreatitis patients. Pancreatitis is an inflammatory process in which pancreatic enzymes are activated leading to autodigestion of the pancreas and adjacent tissues. During pernicious cases, the initial inflammatory response is excessive and can result in tissue injury, organ failure, and death. To counter this inflammation, the immune system initiates a number of inflammatory terminating steps. These steps can include leukocyte apoptosis, anti-inflammatory cytokine production, and anergy $[2,3]$. When patients

*Correspondence: charles.caldwell@uc.edu

'Division of Research, Department of Surgery, University of Cincinnati College of Medicine, Cincinnati, OH 45267-0558, USA

Full list of author information is available at the end of the article become leukopenic and immunosuppressed, they often become susceptible to infections with organisms to which a healthy patient would normally be resistant. Although therapy aimed at blunting the initial overexuberant inflammation may be beneficial if applied early, it may also be detrimental by impairing the host's ability to fight infection that is a common cause of death in these patients. To complicate the potential use of immune-modulating therapy, inflammatory and antiinflammatory processes can occur along different timelines for the varied leukocyte subsets. For example, during immune response, $\mathrm{T}$ lymphocytes might be undergoing apoptosis while neutrophils are primed to produce reactive oxygen species [4]. Thus, during prolonged pancreatitis, leukocyte subsets may be concurrently exhibiting both pro- and anti-inflammatory processes.

As noted above, a potential promising therapy of pancreatitis is modulation of the immune response [5]. This is a difficult undertaking as the host response will be affected by a number of variables, including genetic background, co-morbidities, age, gender, and so on [6]. In addition, the patient's immune response will vary during the course of the disease. Thus, what is needed for immunotherapy to be practical is a rapid, robust measure of the host immune system.

In the manuscript published by Oiva and co-workers [1], the authors determined the signaling profiles of circulating leukocytes isolated from pancreatitis patients using phospho-specific whole blood flow cytometry. This is a powerful new technology that allows for simultaneous single-cell determination of leukocyte subsets using cell surface markers as well as intracellular protein phosphorylation [7]. This work represents a continuation of work published previously [8]. In this earlier work, Oiva and co-workers demonstrated that stimulated monocytes isolated from patients with acute pancreatitis had decreased phosphorylated Erk 1/2, NF-kB, and STAT1 and 3. The authors concluded that these changes could lead to impaired monocyte recruitment as well as increased susceptibility to infections. Here, changes in activated T lymphocyte p38, NF-кB, STAT1 and STAT6 activity was observed that could be interpreted as being 
pro- or anti-inflammatory. Interestingly, they determined that patients' lymphocytes exhibited decreased phosphorylated NF-kB and STAT1 and increased phosphorylation of p38 and STAT6, suggesting a transition from a Th1 to Th 2 phenotype. Additionally, this minimally invasive methodology could be used to generate immune status within 6 hours under ideal conditions. Thus, this methodology represents an essential step prior to targeting the underlying immune response to pancreatitis.

Although beyond the scope of the report, a potentially necessary step after immune status determination, and prior to treatment with inflammatory altering treatments, would be to first treat the whole blood cells isolated from the patients with the potential therapeutic agent and evaluate the immune effector cell response. Phosphospecific flow cytometry could also be used to determine if the leukocytes responded in a way that would be beneficial. Thus, the minimally invasive, relatively quick methodology developed in this paper could be utilized to determine immune status as well as provide a method to test potential therapies.

One unavoidable limitation to the report is that the signaling processes were determined using only peripheral leukocytes. A possibility exists that peripheral leukocytes may not respond similarly to ex vivo stimuli as leukocytes isolated from the inflammatory site(s). When feasible, future studies need to be undertaken to compare the response of peripheral leukocytes to these tissue leukocytes.

Rapid results from whole blood phospho-specific flow cytometry during pancreatitis will allow for immune status determination, likely improve early diagnosis and provide a rational basis for immune targeting therapies. Altogether, this may significantly influence the morbidity and mortality of these patients.

Abbreviations

$\mathrm{NF}$, nuclear factor
Competing interests

The authors declare that they have no competing interests.

\section{Acknowledgements}

This work was supported in part by National Institutes of Health Grants GM44118 and GM55194 (to RSH), GM72760 (to CCC), and the Alan A and Edith LWolff Foundation.

\section{Author details}

'Division of Research, Department of Surgery, University of Cincinnati College of Medicine, Cincinnati, OH 45267-0558, USA. '2Department of Anesthesiology, Washington University School of Medicine, St Louis, MO 63110, USA

Published: 24 January 2011

\section{References}

1. Oiva J, Mustonen H, Kylanpaa ML, Kyhala L, Kuuliala K, Siitonen S, Kemppainen E, Puolakkainen P, Repo H: Acute pancreatitis with organ dysfunction associates with abnormal blood lymphocyte signaling: controlled laboratory study. Crit Care 2010, 14:R207.

2. Bone RC: Sir Isaac Newton, sepsis, SIRS, and CARS. Crit Care Med 1996, 24:1125-1128.

3. Tschop J, Martignoni A, Reid MD, Adediran SG, Gardner J, Noel GJ, Ogle CK, Neely AN, Caldwell CC: Differential immunological phenotypes are exhibited after scald and flame burns. Shock 2009, 31:157-163.

4. Kasten KR, Goetzman HS, Reid MR, Rasper AM, Adediran SG, Robinson CT, Cave CM, Solomkin JS, Lentsch AB, Johannigman JA, Caldwell CC: Divergent adaptive and innate immunological responses are observed in humans following blunt trauma. BMC Immunol 2010, 11:4

5. Hotchkiss RS, Opal S: Immunotherapy for sepsis - a new approach against an ancient foe. N Engl J Med, 363:87-89.

6. Opal SM: Concept of PIRO as a new conceptual framework to understand sepsis. Pediatr Crit Care Med 2005, 6(3 Suppl):S55-60.

7. Krutzik PO, rrish JM, Nolan GP, Perez OD: Analysis of protein phosphorylation and cellular signaling events by flow cytometry: techniques and clinical applications. Clin Immunol 2004, 110:206-221.

8. Oiva J, Mustonen H, Kylanpaa ML, Kyhala L, Alanara T, Aittomaki S, Siitonen S, Kemppainen E, Puolakkainen P, Repo H: Patients with acute pancreatitis complicated by organ failure show highly aberrant monocyte signaling profiles assessed by phospho-specific flow cytometry. Crit Care Med, 38:1702-1708.

doi:10.1186/cc9397

Cite this article as: Caldwell CC, Hotchkiss RS: The first step in utilizing immune-modulating therapies: immune status determination. Critical Care 2011, 15:108. 